\title{
An approach to form the dome shape by 3D laser forming
}

\author{
Q. Nadeem ${ }^{1,2 *}$ and S. J. Na ${ }^{1}$ \\ ${ }^{1}$ Department of Mechanical Engineering, KAIST, Daejeon Yuseong-gu, Korea \\ ${ }^{2}$ COMSATS Inst. of IT, Quaid Venue, The Mall, 47040 Wah Cantt, Pakistan \\ *Corresponding author: sjna@kaist.ac.kr
}

Received August 29, 2012; accepted November 16, 2012; posted online January 21, 2013

\begin{abstract}
Application of a thermal source in non-contact forming of sheet metal is known for some time. Replacement of this thermal source with a laser beam promises the much greater controllability of the process. To date, research focuses on dealing with rectangular plates, and only a few studies are presented for axis-symmetric geometries like circular plates. This study presents the work to get the dish or bowl shape by an initially flat circular plate. Two different scanning strategies circular and radial are attempted to get the desired dish shape. Following the unexpected distortion throughout the plate, a second series of experiments are conducted on a wide range of specimen geometries. An interesting phenomenon is observed. It is suggested that homogeneous dissemination of heat along with combined form of both of the scanning strategies, could have more potential to form dome shape.
\end{abstract}

OCIS codes: $140.0140,160.0160$.

doi: 10.3788/COL201311.021402.

Forming techniques consist of metal working processes in which the material is shaped in solid state by plastic deformation. Mainly, there are two classifications of forming techniques called bulk forming and sheet forming. Rolling, extrusion and forging are part of bulk forming. On the other hand, bending and contouring etc. are common processes of sheet forming. Conventional bending of sheet metal by using punch and die system requires hard tooling, external forces and has spring back action. The idea of using lasers for bending the sheet metal was first conceived by Kitamura ${ }^{[1]}$. Laser forming is a relatively young and fast-growing field of laser material processing. Moshaiov et al. ${ }^{[2]}$ have shown that the process is similar to the well-established torch flame bending used on large sheet material in the ship building industry but as a great deal, more control of the final product can be realized. Compared with conventional forming techniques, laser forming of sheet metal components and tubes does not require hard tooling or external forces. Such advantage can increase process flexibility and reduce the cost of the forming process when low-to-medium-production volume is concerned ${ }^{[3-5]}$.

In recent years, laser-forming techniques have been extensively investigated. The empirical, analytical and FEM tools ${ }^{[6,7]}$ have been used to predict the distortions and relation between bending angle and process parameters like power of laser beam, the speed at which laser beam scans the sheet metal, laser beam spot diameter and number. of passes at fixed path etc. However, the results were highly case-dependent.

The scanning path of the laser is dependent on the desired shape. In the simplest case, the path may be a point ${ }^{[8]}$, in other cases the path may be a straight line across the whole part ${ }^{[9-12]}$ and, for spatially formed parts and extrusions the path would be very sophisticated radial and tangential lines ${ }^{[13-15]}$. Nevertheless, it is suggested that laser forming is a process capable of producing complex shapes such as sine, dish, saddle, pillow, cone and screw. Most of the research to date has focused on laser forming along linear irradiation paths and over rectangular plates. However, in order to take the process closer to real life applications, an investigation into other possible scanning patterns for a class of geometrical shapes including circular plate becomes a necessity. Different scanning strategies have to be developed for the production of complex shaped parts (e.g. a spherical dome). The certain deformation can be obtained if laser beam is scanning the sheet several times along the same path. Edwardson et al. ${ }^{[16]}$ studied the forming process of the saddle shape parts by using five different scanning strategies. Results show that the warped deformation occurs because of the dissymmetry of laser irradiations. Váquez-Ojeda et al. ${ }^{[17]}$ investigated a few other scanning strategies, like zigzag, squared, and stepped scan lines and claimed that round corners of bend are achievable. A heating strategy was experimentally investigated by a research group ${ }^{[18]}$ to produce a bowl-shape. However, the quality of the shape obtained could not be fully described due to the difficulty of the measurement of micro-size displacement. Hennige ${ }^{[19]}$ proposed a heating path strategy for ring and circle segments, but the range of segment angle was limited. Moreover, the waviness of circular plates was not fully addressed.

Currently, attempts are being made to generate more complex shapes. Therefore, for the production of complex spatially curved shapes, scanning strategies based on curved scanning paths can be used, alone, or along with the linear scanning paths. We have concluded that laser forming of the circular shape specimens with curved scanning paths have different deformation behaviors than rectangular shapes. Thus, we attempt to extend our former study ${ }^{[20,21]}$ towards full circular plate for bending it into the shape of spatially curved bowl. The deformation pattern and pertinent hurdles to get the desired bowl shape have been recognized. The stated geometry was subjected mainly to concentric scanning paths, though, other scanning strategies like radially-linear, have also been explored, with the aim of identifying the deficits of each scanning strategy and proposing solution for the same. These scanning paths are more likely to form 
three-dimensionally curved components like bowl, dome, and cup shapes.

Figure 1 shows the schematic diagram of circular heating process used for study. However, radially-linear heating paths i.e. along the radial direction of specimen (not shown in Fig. 1) were also used. Figure 2 shows the experimental setup. Fiber laser has maximum power of $100 \mathrm{~W}$ and motion assistant was used to control the 3 -axis CNC table. In all experiments, the defocused Gaussian-mode laser beam had constant power of $80 \mathrm{~W}$, while the beam's moving velocity and diameter were 720 $\mathrm{mm} / \mathrm{min}$ and $1 \mathrm{~mm}$, respectively. Time delay between any two consecutive passes was 90 seconds in the case of multiple numbers of irradiations $(\mathrm{N})$. A laser displacement sensor (Model: LB-081, III a) was used to measure bending angles of the deformed plates. Hereby a basic part geometry, which can be thought as a circular plate having two radii ( $R_{\mathrm{o}}$ is outer radius and $R_{\mathrm{i}}$ is inner radius) was selected. The specimens have outer radius of $50 \mathrm{~mm}$ and inner radius of $10 \mathrm{~mm}$. Thickness of all specimens was $0.8 \mathrm{~mm}$ and to enhance laser absorption by the work-piece, graphite coating ${ }^{[22]}$ is applied to the initial flat surface. For instance, "segment angle $(\beta)$ " is defined as total central angle of a specimen. The numerous plates with different segment angles $(\beta)$ like $210^{\circ}$, $240^{\circ}$, and $330^{\circ}$ were used. The reason for this will be discussed in the subsequent section. After experiments, bend angle was measured at various positions along the particular circular/radial heating path. As a general rule the minimum number of positions for measurement along the heating path were three. To compensate the edge effects, the bending angle was not measured at or very close to the edges of specimens. The values of bending angles shown in the manuscript are taken as root mean square (RMS) average of measured angles at different locations. A specially designed jig was used which can rotate about $z$-axis, so that measurements can be facilitated accurately. All specimens were made of common engineering low-carbon steel.

Nadeem et al. ${ }^{[23]}$ handled the inverse problem for quarter-circular $\left(\beta=90^{\circ}\right)$ and half-circular plates $\left(\beta=180^{\circ}\right)$; designing process parameters and scanning path planning, given a desired shape. They found that concentric paths alone were applicable enough for these geometries. Therefore, this study primarily attempted to form the initially flat circular $\left(\beta=360^{\circ}\right)$ plate into the dome shape. Though, similar to other studies ${ }^{[12,19]}$ empirical based

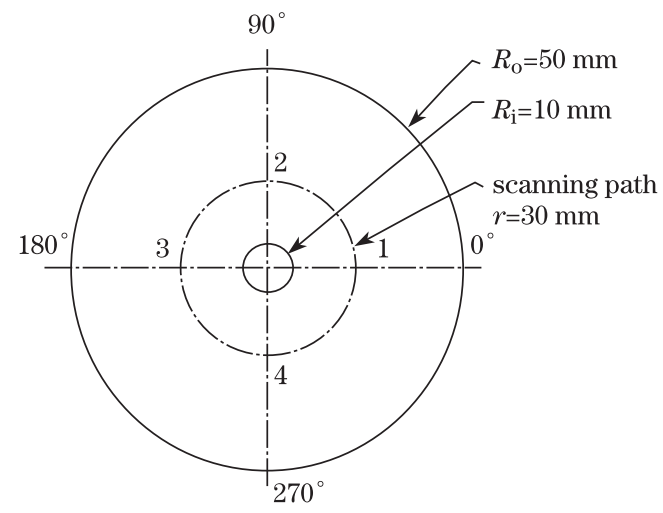

Fig. 1. Schematic of the circular scanning path.

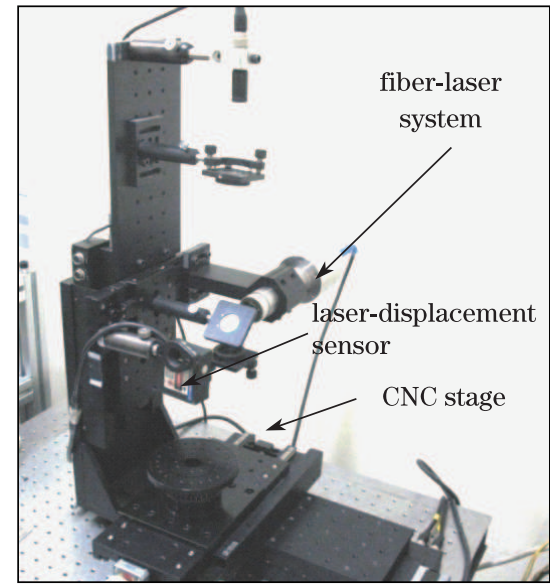

Fig. 2. Experimental setup.

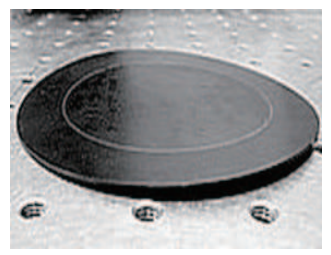

(a)

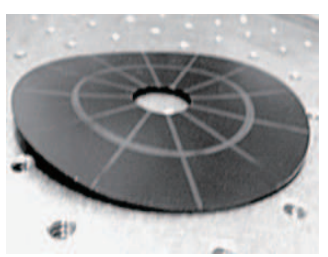

(b)
Fig. 3. Distortion in plate after experiments by (a) concentric and (b) combined radial scanning paths.

scanning strategies were executed experimentally. Figure 3 (a) shows result of a fundamental experiment with arbitrary concentric path. Surprisingly, an unwanted distortion occurred throughout the plate. This initiated the need to ascertain the reason in a well fashion.

As we already found ${ }^{[20]}$ that deformation behavior was fairly symmetrical for plate with $\beta$ up until $180^{\circ}$, so in order to find the threshold point at which deformation transits to unsymmetrical one; experiments were extended to specimens of $180^{\circ} \leqslant \beta \leqslant 360$. Heating conditions were identical as preceding ones. Besides, the heating path radius was kept constant to $30 \mathrm{~mm}$ for this investigation. Figure 4 shows the whole deformation behavior for previous range ${ }^{[20]}\left(0\right.$ to $\left.180^{\circ}\right)$ and present study's range $\left(180^{\circ}\right.$ to $\left.330^{\circ}\right)$ of $\beta$.

Likewise, Fig. 5 shows the final deformed plates after experiments. It is very conclusive to state that unwanted distortion is not so far obvious even for $\beta$ up to $330^{\circ}$. Before going to subsequent section, it is handy to define, (Fig. 5(e)) the "free edges" of specimen i.e. the edges which are unrestricted to expand / shrink during heating and cooling cycles, at the start and end of laser scanning process respectively. For a certain plate, for instance with $\beta=330^{\circ}$, it is clear that angular gap between these free edges is $30^{\circ}$.

After these results, to find out the threshold value at which unwanted distortion initiated, further experiments with possibly appropriate linear gap between two free edges were opted. Figure 6 shows the results of these experiments. Figure 6(a) shows the plates of radially linear uniform gap of $3 \mathrm{~mm}$ between free edges. The attentive monitoring of these experiments was found to be useful for 3D laser forming of dish-shape. It is remarkable to find that initial gap i.e. $3 \mathrm{~mm}$ between both free edges, 


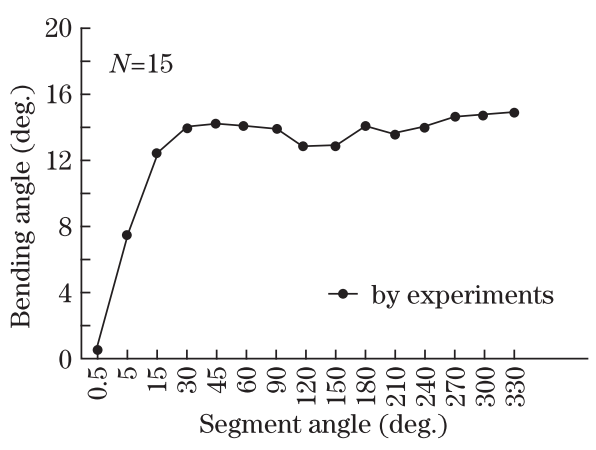

Fig. 4. Deformation behaviors of specimens with $\beta$ up to $330^{\circ}$

is diminishing, non-equally along the radial direction of specimen. Two sets of plates were taken, one with initial gap of $3 \mathrm{~mm}$ while the other with $1.5 \mathrm{~mm}$. Figure 6 (a) shows initial plate (gap $=3 \mathrm{~mm}$ ) before the laser beam scans the plate $(N=0)$. When the laser is passed several times along the same path, interestingly the gap between free edges started to decrease, as shown by Figs. 6(b)-(e). Moreover, this effect was more obvious near the region of outer periphery. In other words, intensity of this "gap effect' is increasing from inner radius to outer radius and consequently the gap around inner region is wider than the outer region as revealed by Figs. 6(b) and (d). The final deformation's nature and amount of total bend angle after cooling is obtained, among others, from the geometrical conditions that includes restraining forces due to the moment of inertia during cooling. Therefore this effect is attributed to the changing mechanical restraint, along radial direction, which hinders the free thermal expansion with distance from the edge of the metal sheet. This suggests that the region which has lower rigid constraint is being extended.

It confirmed the similar observation reported by Chen et al. ${ }^{[24,25]}$. They studied the laser curve bending of Ti$6 \mathrm{Al}-4 \mathrm{~V}$ alloy steel sheet metal, for rectangular sheet with curved scanning path, and showed that 3D deformation occurs only on one side of a scanning path, along which, the rigid constraint is relatively lower, and on this side, the sheet is extended and thinned slightly.

Each scanning pass results into the accumulation of the stated gap effect. After some more consecutive passes the edges near to outer periphery, were at verge to overlap, shown in Figs. 6(b) and (d). At this locus, further irradiation pass triggered the unwanted distortion in the plate; Figs. 6(c) and (e). Comprehensibly, in case of full-circular plate which exclusively does not have any gap or free edges at all; the same phenomenon influences the plate at very early stages of process. Another perspective could be because of non-equal amount of surrounding material along the scanning path. On the other hand, rectangular shape plate has uniform distribution of surrounding material while using linear scanning paths. Hence no such effect was observed in rectangular case. It implies that with the intention to utilize the concentric paths for getting the dish shape, possible solution is to heat the plate in such a way that results into the uniform distribution of temperatures throughout the scanning path. And this in turn could develop stresses that likely are favorable for symmetric deformations along the path.
Figure 3(b) shows the result of experiment using such radial irradiation paths. In order to handle the issue, experiments were conducted over the plate having an initial gap of $3 \mathrm{~mm}$ between free edges.

Figure 7 shows the result of experiment on a fullcircular plate and point towards the potential of radial type heating paths, to get the desired shape. It is noticeable that full-length radial heating paths i.e. equivalent to net radius of plate $40 \mathrm{~mm}$, would also cause some gap effect in the plate. Nevertheless, the gap effect for total deformation is not as much as of circular paths. The plate has large symmetrical bending than curved paths. It

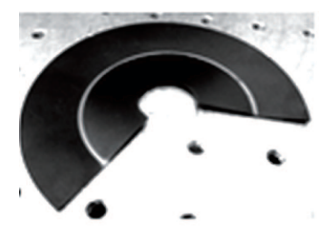

(a)

(c)

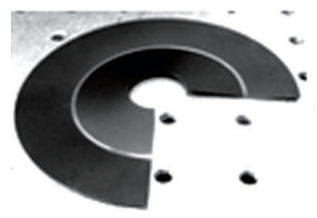

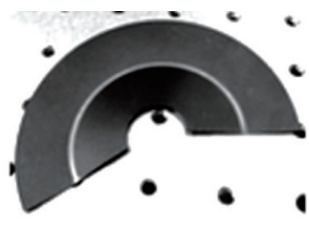

(b)

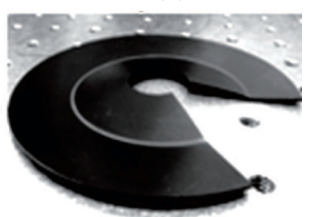

(d)

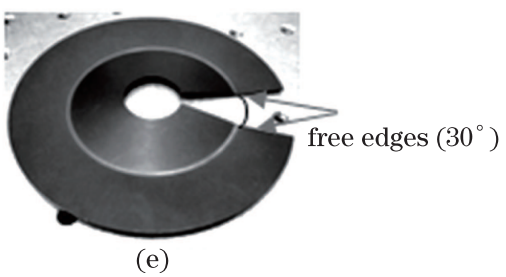

Fig. 5. Deformed plates with (a) $210^{\circ}$, (b) $240^{\circ}$, (c) $270^{\circ}$, (d) $300^{\circ}$, and (e) $330^{\circ}$, respectively; path radius is $30 \mathrm{~mm}$ and $N=15$.

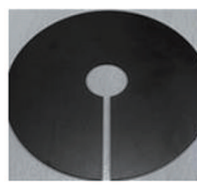

(a) $N=0$

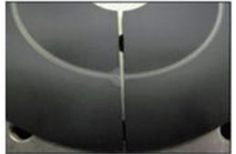

(d) $N=7$

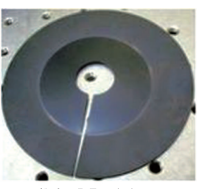

(b) $N=11$

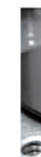

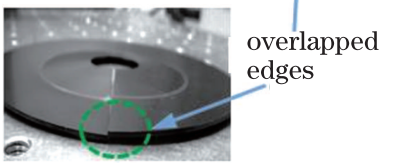

(e) $N=11$
Fig. 6. Stages of distortion, path raduis is $30 \mathrm{~mm}$, increasing number of scanning passes $(N)$; free edges come towards each other nonqually along radial drection, (a-c) initial gap between free edges is $3 \mathrm{~mm}$, (d, e) initial gap is $1.5 \mathrm{~mm}$,

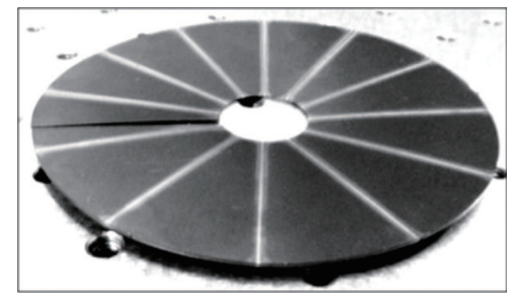

Fig. 7. Result of arbitrarily chosen radial irradiation paths for plate (gap is $3 \mathrm{~mm}$ ). 
revealed that used strategy caused very less unwanted distortions in the plate. The material can relax through the free edges. This demonstrates potential of radial type irradiation path for this class of geometry.

Geiger et al. ${ }^{[26]}$ reported that the important mechanism, which offers the possibility of producing threedimensionally curved components similar to the deep drawn parts, is the upsetting mechanism. For this mechanism the irradiation parameters are chosen in such a way that a heat penetration as homogeneous as possible of the component in direction perpendicular to the sheet surface is obtained. Therefore, in this context, one possible solution to non-equal surrounding material could be use of radially linear irradiation paths, with varying length and combined (circular and radial) type paths in such a way that heat disseminates throughout the plate homogeneously.

In conclusion, the study presented above is opening new dimensions in understanding the laser forming process for producing three-dimensionally curved components like dish, cup and dome shapes. A series of investigation are carried out to analyze the influence of the curved irradiations and radial irradiations for circular geometry. Both curved and radial scanning strategies were experienced for getting the desired dish shape from initial flat circular plate. Radial scanning paths caused less unwanted distortion than curved paths. Nonetheless, it is found, for both scanning strategies, that non-uniform distribution of surrounding material causes the unwanted distortion throughout the plate. This effect is attributed to the geometrical effect and thus changing mechanical restraint. Therefore, the key is to heat the plate in such a way that the heat penetrates homogeneously throughout the plate. Either use of combination of curved and radially linear paths or only radially-linear paths with varying length might be possible solutions.

This work was supported by the Higher Education Commission (HEC) of Pakistan.

\section{References}

1. N. Kitamura, JWES-TP-8302 359 (1983).

2. A. Moshaiov and W. Vorus, J. Ship Research 31, 269
(1987).

3. H. Frackiewicz, Fabtech International, Illinois 723 (1993).

4. M. Geiger, Annals of the CIRP 43, 563 (1994).

5. D. F. Walczyk and S. Vittal, J. Manuf. Proc. 2, 258 (2000).

6. Z. Ji and S. Wu, J. Mater. Process. Technol. 74, 89 (1998).

7. A. K. Kyrsanidi, T. B. Kermanidis, and S. G. Pantelakis, J. Mater. Process. Technol. 104, 94 (2000).

8. J. Widlaszewski, in Proceedings of the LANE 569 (2001).

9. Y. Namba, in Proceedings of International Conference on Lasers 403 (1985).

10. K. Scully, J. Ship Production 3, 237 (1987).

11. M. Geiger, H. Arnet, and F. Vollertsen, in Proceedings of the LANE 81 (1994).

12. J. Magee, K. G. Watkins, W. M. Steen, N. J. Clader, J. Sidhu, and J. Kirby, in Proceedings of ICALEO'97 156 (1997).

13. T. Hennige, in Proceedings of the LANE 409 (1997).

14. J. Magee, K. G. Watkins, and T. Hennige, in Proceedings of ICALEO 77 (1999).

15. K. Masubuchi, in Proceedings of LAMP 939 (1992).

16. S. P. Edwardson, K. G. Watkins, G. Dearden, and J. Magee, in Proceedings of 3rd International Conference on Laser Assisted Net Shaping Erlangen 559 (2001).

17. C. Váquez-Ojeda and J. Ramos-Grez, J. Mater. Process. Technol. 209, 2641 (2009).

18. J. Magee, K. G. Watkins, and T. Hennige, in Proceedings of ICALEO 77 (1999).

19. T. Hennige, J. Mater. Process. Technol. 103, 2 (2001).

20. Q. Nadeem and S. J. Na, Chin. Opt. Lett. 9, 051402 (2011).

21. Q. Nadeem, W. J. Seong, and S. J. Na, in Proceedings of Autumn Annual Conference of KWJS 22 (2009).

22. J. Kim and S. J. Na, Opt. Laser Technol. 35, 605 (2003).

23. Q. Nadeem, W. J. Seong, and S. J. Na, Chin. Opt. Lett. 10, 021405 (2012).

24. D. J. Chen, S. C. Wu, and M. Q. Li, J. Mater. Process. Technol. 152, 62 (2004).

25. P. Zhang, B. Guo, D. Shan, and Z. Ji, J. Mater. Process. Technol. 184, 157 (2007).

26. M. Geiger and F. Vollertsen, Leichtbaustrukturen Und Leichte Bauteile. 1080, 293 (1994). 